\title{
Application of Sumudu Transform in Generalized Fractional Reaction-Diffusion Equation
}

\author{
Badr Alkahtani $^{1}$ - Vartika Gulati ${ }^{2}$ - Adem Kılıçman ${ }^{3}$
}

\begin{abstract}
In this paper we investigate the solution of a generalized nonlinear fractional reaction diffusion equation by the application of Sumudu Transform.
\end{abstract}

Keywords Generalized fractional derivatives - Fractional diffusion equation · Generalized Mittag-Leffler function · Sumudu transform and Fourier transform

Mathematics Subject Classification Primary 26A33 - Secondary 33E12

\section{Introduction}

Reaction-diffusion systems (RDS) have been extensively used in the study of selforganization phenomena in physics, biology, chemistry, ecology, etc. see Smoller [1], Grindrod [2], Gilding and Kersner [3], and Wilhelmsson and Lazzaro [4]. These systems show that diffusion can produce the spontaneous formation spatiotemporal patterns. For details, refer to the work of Nicolis and Pri-Gogine [5]. A general model for reaction diffusion systems is discussed by Henry and Wearne [6,7]. For the some applications of the Laplace decomposition method for solving linear and nonlinear fractional diffusion-wave equations, see [5,8-10].

\footnotetext{
$凶$ Adem Kılıçman

akilic@upm.edu.my

Badr Alkahtani

alhaghog@googlemail.com

Vartika Gulati

vartikagulati82@gmail.com

1 King Saud University, Riyadh, Saudi Arabia

2 Department of Mathematics, University of Rajasthan, Jaipur 302055, Rajasthan, India

3 Department of Mathematics, University Putra Malaysia, 43400 UPM Serdang, Selangor, Malaysia
} 
A similar approach was recently used by Manne et al. [11] to investigate effects on the propagation of nonlinear wave fronts. The simplest reaction-diffusion models can be described by an equation

$$
\frac{\partial N}{\partial t}=D \frac{\partial^{2} N}{\partial x^{2}}+\gamma F(N)
$$

where $\mathrm{D}$ is the diffusion coefficient and $\mathrm{F}(\mathrm{N})$ is a nonlinear function representing reaction kinetics. For $\mathrm{F}(\mathrm{N})=\gamma \mathrm{N}(1-\mathrm{N})$, Eq. (1) reduces to Fisher Kolmogorov equation and for $\mathrm{F}(\mathrm{N})=\gamma \mathrm{N}\left(1-N^{2}\right)$, it reduces to the real Ginsburg-Landau equation. A generalization of (1) has been considered by Manne et al. [11] in the form

$$
\frac{\partial^{2} N}{\partial t^{2}}+a \frac{\partial N}{\partial t}=v^{2} \frac{\partial^{2} N}{\partial x^{2}}+\xi^{2} N(x, t)
$$

where $\xi$ indicates the strength of the nonlinearity of the system. Saxena et al. [12] generalized above equation using time-fractional derivatives. In this work, we present a straight forward method for the systematic derivation of the solution of nonlinear reaction-diffusion equations that is connected with nonlinear waves, which is also more general than the Eq. (2).

\section{Definitions and Preliminaries}

Definition 2.1 Over the set of functions,

$$
A=\left\{f(t)\left|\exists M, \tau_{1}, \tau_{2}>0,\right| f(t) \mid<M e^{\frac{|t|}{\tau_{i}}}, \quad \text { if } t \in(-1)^{j} \times[0, \infty)\right\}
$$

the Sumudu Transform is

$$
G(u)=S[f(t)]=\int_{0}^{\infty} f(u t) e^{-t} d t, \quad u \in\left(-\tau_{1}, \tau_{2}\right) .
$$

For further detail and properties of this transform, see [13-18]. We will establish the following results which are directly applicable in the analysis of reaction diffusion systems.

Definition 2.2 A generalization of the Mittag-Leffler function, see [19].

$$
E_{\alpha}(z)=\sum_{k=0}^{\infty} \frac{z^{k}}{\Gamma(k \alpha+1)}
$$

where $\Re(\alpha)>0 ; z \in \mathbb{C}$.

A generalization of Mittag-Leffler function $E_{\alpha}(z)$ of (5) is defined and studied by Wiman [20], as follows:

$$
E_{\alpha, \beta}(z)=\sum_{k=0}^{\infty} \frac{z^{k}}{\Gamma(\alpha k+\beta)}
$$

where $\alpha, \beta \in R(\alpha)>0 ; \quad R(\beta)>0 ; \quad z \in C$

A generalization of Mittag-Leffler function $E_{\alpha, \beta}(z)$ of (6) is introduced by Prabhakar [21], as follows:

$$
E_{\alpha, \beta}^{\gamma}(z)=\sum_{k=0}^{\infty} \frac{\gamma_{k}}{\Gamma(\alpha k+\beta)} \frac{z^{k}}{k !}
$$


where $\alpha, \beta \in C ; R(\alpha)>0 ; R(\beta)>0 ; z \in C, \gamma_{k}$ denotes the familiar Pochhammer symbol or the shifted factorial.

Recently, a remarkably large family of Generalized Riemann-Liouville fractional derivatives of order $\alpha(0<\alpha<1)$ and type $\beta(0 \leq \beta \leq 1)$ was introduced as follows [22,23].

Definition 2.3 The right-sided fractional derivative $\left(D_{a+}^{\alpha, \beta}\right)$ and the left sided fractional derivative $\left(D_{a-}^{\alpha, \beta}\right)$ of order $\alpha(0<\alpha<1)$ and type $\beta(0 \leq \beta \leq 1)$ with respect to $\mathrm{x}$ are defined by

$$
\left(D_{a \pm}^{\alpha, \beta} f\right)(x)=\left( \pm I_{a \pm}^{\beta(1-\alpha)} \frac{d}{d x}\left(I_{a \pm}^{(1-\beta)(1-\alpha)} f\right)\right)(x)
$$

whenever the right hand side of (8) exist. This generalization yields the classical RiemannLiouville fractional derivative operator when $\beta=0$ moreover for $\beta=1$, it gives the fractional derivative operator introduced by Liouville [24], which is often attributed to Caputo now-adays and which should more appropriately be referred to as the Liouville-Caputo fractional derivative, see [25].

The fractional derivative operator $\left(D_{a \pm}^{\alpha, \beta}\right)$ can be rewritten in the following form :

$$
\left(D_{a \pm}^{\alpha, \beta}\right)(x)=\left( \pm I_{a \pm}^{\beta(1-\alpha)}\left(D_{a \pm}^{\alpha+\beta-\alpha \beta} f\right)\right)(x) .
$$

Definition 2.4 Caputo [25] introduced fractional derivative in the following form

$$
\begin{aligned}
{ }_{0} D_{t}^{\alpha} f(t) & =\frac{1}{\Gamma(m-\alpha)} \int_{0}^{t} \frac{f^{m}(\tau)}{(t-\tau)^{\alpha-m+1}} d \tau, \quad m-1<\alpha<m, R(\alpha)>0 \\
& =\frac{d^{m} f}{d t^{m}}, \quad \text { if } \quad \alpha=m .
\end{aligned}
$$

Definition 2.5 The Weyl fractional operator defined by

$$
{ }_{-\infty} D_{x}^{\mu} f(t)=\frac{1}{\Gamma(n-\mu)} \frac{d^{n}}{d t^{n}} \int_{-\infty}^{t} \frac{f(u)}{(t-u)^{\mu-n+1}} d u,
$$

where $n=[\mu]$ is an integral part of $\mu>0$.

Its Fourier transform is given by Metzler and Klafter [26].

$$
F\left\{{ }_{-\infty} D_{t}^{\mu} f(t)\right\}=(i k)^{\mu} f^{*}(k)
$$

where $f^{*}(k)$ denotes the Fourier Transform of the function $f(x)$. Further modification of result (12) is given Metzler and Klafter [27].

$$
F\left\{{ }_{-\infty} D_{x}^{\mu} f(x)\right\}=-|k|^{\mu} f^{*}(k) .
$$

\section{Main Results}

We will establish the following results which are directly applicable in the analysis of reaction diffusion systems. 
Lemma 3.1 In the complex plane $C$, for any $R\{\alpha\}>0, R\{\beta\}>0$ and $\omega \in C$

$$
S^{-1}\left[u^{\gamma-1}\left(1-\omega u^{\beta}\right)^{-\delta}\right]=t^{\gamma-1} E_{\beta, \gamma}^{\delta}\left(\omega t^{\beta}\right) .
$$

We can prove the result in another way

$$
S\left[t^{\gamma-1} E_{\beta, \gamma}^{\delta}\left(\omega t^{\beta}\right)\right]=\int_{0}^{\infty} e^{-t}(u t)^{\gamma-1} E_{\beta, \gamma}^{\delta}\left(\omega(u t)^{\beta}\right) d t .
$$

By using Eq. (7)

$$
u^{\gamma-1} \sum_{n=0}^{\infty} \frac{(\delta)_{n}\left(\omega u^{\beta}\right)^{n}}{n !}=u^{\gamma-1}\left(1-\omega u^{\beta}\right)^{-\delta} .
$$

By applying inverse Sumudu Transform, we get our required result.

Lemma 3.2 The complex plane $C$, for any $R\{\alpha\}>0, R\{\beta\}>0$

$$
S^{-1}\left[\frac{1}{u\left(u^{-\alpha}+a u^{-\beta}+b\right)}\right]=\sum_{r=0}^{\infty}(-b)^{r} t^{\alpha(r+1)-1} E_{\alpha-\beta, \alpha(r+1)}^{r+1}\left[-a t^{\alpha-\beta}\right] .
$$

Lemma 3.3 The complex plane $C$, for any $R\{\alpha\}>0, R\{\beta\}>0$

$$
S^{-1}\left[\frac{u^{-1+\alpha_{1}(1-\alpha)}}{u^{-\alpha}+a u^{-\beta}+b}\right]=\sum_{r=0}^{\infty}(-b)^{r} t^{\left(r+1-\alpha_{1}\right) \alpha+\alpha_{1}-1} E_{\alpha-\beta,\left(r+1-\alpha_{1}\right) \alpha+\alpha_{1}}^{r+1}\left(-a t^{\alpha-\beta}\right) .
$$

Lemma 3.4 The complex plane $C$, for any $R\{\alpha\}>0, R\{\beta\}>0$

$$
S^{-1}\left[\frac{a u^{-1 \alpha_{1}+(1-\beta)}}{u^{-\alpha}+a u^{-\beta}+b}\right]=\sum_{r=0}^{\infty} a \cdot(-b)^{r} t^{+\alpha_{1}(1-\beta)+(r+1) \alpha-1} E_{\alpha-\beta, \alpha_{1}(1-\beta)+(r+1) \alpha}^{r+1}\left(-a t^{\alpha-\beta}\right) .
$$

Lemma 3.5 The Sumudu transform of the generalized fractional derivative is given by

$$
S\left[{ }_{0} D_{t}^{\alpha, \beta} f(x)\right]=S\left( \pm I_{a \pm}^{\beta(1-\alpha)} \frac{d}{d x}\left(I_{a \pm}^{(1-\beta)(1-\alpha)} f\right)\right)(x) .
$$

By using Laplace Sumudu duality, see [14-17,28], we get the following result

$$
u^{-\alpha} S[f(x)] u-\sum_{k=0}^{n-1} u^{k-n+\beta(n-\alpha)} \frac{d^{k}}{d x^{k}}\left(I_{0}^{(1-\beta)(n-\alpha)} f\right)(0+) .
$$

Theorem 3.1 Consider the fractional reaction diffusion equation

$$
\begin{aligned}
& { }_{0} D_{t}^{\alpha, \alpha_{1}} N(x, t)+a_{0} D_{t}^{\beta, \alpha_{1}} N(x, t)=v^{2}{ }_{-\infty} D_{x}^{\gamma} N(x, t)+\xi^{2} N(x, t)+\psi(x, t) \\
& 0<\alpha<1,0<\beta<1,0<\alpha_{1} \leq 1
\end{aligned}
$$

with initial conditions

$$
\begin{aligned}
& I_{t}^{\left(1-\alpha_{1}\right)(1-\alpha)} N(x, 0)=\phi_{1}(x) \\
& I_{t}^{\left(1-\alpha_{1}\right)(1-\beta)} N(x, 0)=\phi_{2}(x)
\end{aligned}
$$


where $v$ is a diffusion coefficient, $\psi$ is constant which describes the nonlinearity in the system, and is a nonlinear function for reaction kinetics then there holds the following formula:

$$
\begin{aligned}
N(x, t)= & \sum_{r=0}^{\infty} \frac{(-b)^{r}}{\sqrt{2 \pi}} \int_{-\infty}^{\infty} t^{\left(r+1-\alpha_{1}\right) \alpha+\alpha_{1}-1} \phi_{1}^{*}(k) \exp (-i k x) \\
& \times E_{\alpha-\beta,\left(r+1-\alpha_{1}\right) \alpha+\alpha_{1}}^{r+1}\left(-a t^{\alpha-\beta}\right) d k \\
& -\sum_{r=0}^{\infty} a \frac{(-b)^{r}}{\sqrt{2 \pi}} \times \int_{-\infty}^{\infty} t^{\alpha_{1}(1-\beta)+(r+1) \alpha-1} \phi_{2}^{*}(k) \exp (-i k x) \\
& \times E_{\alpha-\beta, \alpha_{1}(1-\beta)+(r+1) \alpha}^{r+1}\left(a t^{\alpha-\beta}\right) d k \\
& +\sum_{r=0}^{\infty} \frac{(-b)^{r}}{\sqrt{2 \pi}} \int_{0}^{\mathrm{t}} \tau^{\alpha(r+1)-1} \int_{-\infty}^{\infty} \psi^{*}(k, t-\tau) \exp (-i k x) E_{\alpha-\beta, \alpha(r+1)}^{r+1} \\
& \left(-a \tau^{\alpha-\beta}\right) d k d \tau \quad
\end{aligned}
$$

where $\alpha>\beta$ and $E_{\beta, \gamma}^{\delta}(z)$ is the generalized Mittag-Leffler function, defined in (6) and $b=v^{2}|k|^{\gamma}-\xi^{2}$.

Proof Applying the Sumudu Transform with respect to the time variable t, we find,

$$
\begin{gathered}
u^{-\alpha} \bar{N}(x, u)-u^{-1+\alpha_{1}(1-\alpha)}\left[I_{0}^{\left(1-\alpha_{1}\right)(1-\alpha)} N(x, 0)\right]+a u^{-\beta} \bar{N}(x, u) \\
-a u^{-1+\alpha_{1}(1-\beta)}\left[I_{t}^{\left(1-\alpha_{1}\right)(1-\beta)} N(x, 0)\right] \\
=v^{2}{ }_{-\infty} D_{x}^{\gamma} \bar{N}(x, u)+\xi^{2} \bar{N}(x, u)+\bar{\psi}(x, u)
\end{gathered}
$$

Using initial conditions, Eq. (22) can be written as

$$
\begin{aligned}
& u^{-\alpha} \bar{N}(x, u)-u^{-1+\alpha_{1}(1-\alpha)} \phi_{1}(x)+a u^{-\beta} \bar{N}(x, u)-a u^{-1+\alpha_{1}(1-\beta)} \phi_{2}(x) \\
& \quad=v_{-\infty}^{2} D_{x}^{\gamma} \bar{N}(x, u)+\xi^{2} \bar{N}(x, u)+\bar{\psi}(x, u)
\end{aligned}
$$

Taking Fourier transform of above Eq. (23) we obtain

$$
\begin{aligned}
& u^{-\alpha} \bar{N}^{*}(k, u)-u^{-1+\alpha_{1}(1-\alpha)} \phi_{1}^{*}(x)+a u^{-\beta} \bar{N}^{*}(k, u)-a u^{-1+\alpha_{1}(1-\beta)} \phi_{2}^{*}(x) \\
& =v^{2}|K|^{\gamma} \bar{N}^{*}(k, u)+\xi^{2} \bar{N}^{*}(k, u)+\bar{\psi}^{*}(k, u)
\end{aligned}
$$

Solving for $\bar{N}^{*}(k, u)$

$$
\bar{N}^{*}(k, u)=\frac{u^{-1+\alpha_{1}(1-\alpha)} \phi_{1}^{*}(k)}{u^{-\alpha}+a u^{-\beta}+b}-\frac{u^{-1+\alpha_{1}(1-\beta)} \phi_{2}^{*}(k)}{u^{-\alpha}+a u^{-\beta}+b}+\frac{\bar{\psi}^{*}(k, u)}{u^{-\alpha}+a u^{-\beta}+b}
$$

where $b=v^{2}|k|^{\gamma}-\xi^{2}$, Inverting the Sumudu transform with the help of Eqs. (18) and (21). 


$$
\begin{aligned}
N^{*}(k, t)= & \sum_{r=0}^{\infty}(-b)^{r} t^{\left(r+1-\alpha_{1}\right) \alpha+\alpha_{1}-1} E_{\alpha-\beta,\left(r+1-\alpha_{1}\right) \alpha+\alpha_{1}}^{r+1}\left(-a t^{\alpha-\beta}\right) \phi_{1}^{*}(k) \\
& -\sum_{r=0}^{\infty} a \cdot(-b)^{r} t^{\alpha_{1}(1-\beta)+(r+1) \alpha-1} E_{\alpha-\beta, \alpha_{1}(1-\beta)+(r+1) \alpha}^{r+1}\left(-a t^{\alpha-\beta}\right) \phi_{2}^{*}(k) \\
& +\sum_{\mathrm{r}=0}^{\infty}(-b)^{r} \int_{0}^{\mathrm{t}} \psi^{*}(k, t-\tau) \tau^{\alpha(\mathrm{r}+1)-1} E_{\alpha-\beta, \alpha(r+1)}^{r+1}\left(-a \tau^{\alpha-\beta}\right) d \tau
\end{aligned}
$$

on using the convolution theorem of Sumudu transform (see $[14,16,17]$ ). Now by applying inverse Fourier transform we get the required result of reaction diffusion equation in terms of generalized Mittag-Leffler function.

$$
\begin{aligned}
N(x, t)= & \sum_{r=0}^{\infty} \frac{(-b)^{r}}{\sqrt{2 \pi}} \int_{-\infty}^{\infty} t^{\left(r+1-\alpha_{1}\right) \alpha+\alpha_{1}-1} \phi_{1}^{*}(k) \exp (-i k x) \\
& \times E_{\alpha-\beta,\left(r+1-\alpha_{1}\right) \alpha+\alpha_{1}}^{r+1}\left(-a t^{\alpha-\beta}\right) d k \\
& -\sum_{r=0}^{\infty} a \frac{(-b)^{r}}{\sqrt{2 \pi}} \cdot \int_{-\infty}^{\infty} t^{\alpha_{1}(1-\beta)+(r+1) \alpha-1} \phi_{2}^{*}(k) \exp (-i k x) \\
& \times E_{\alpha-\beta, \alpha_{1}(1-\beta)+(r+1) \alpha}^{r+1}\left(-a t^{\alpha-\beta}\right) d k \\
& +\sum_{\mathrm{r}=0}^{\infty} \frac{(-b)^{r}}{\sqrt{2 \pi}} \int_{0}^{\mathrm{t}} \tau^{\alpha(r+1)-1} \int_{-\infty}^{\infty} \psi^{*}(k, t-\tau) \exp (-i k x) E_{\alpha-\beta, \alpha(r+1)}^{r+1} \\
& \left(-a \tau^{\alpha-\beta}\right) d k d \tau
\end{aligned}
$$

In particular, if we set $\alpha_{1}=1$ we get fractional diffusion reaction equation in following form studied by Gupta and Sharma [10].

$$
\begin{gathered}
{ }_{0} D_{t}^{\alpha} N(x, t)+a_{0} D_{t}^{\beta_{1}} N(x, t)=v^{2}{ }_{-\infty} D_{x}^{\gamma} N(x, t)+\xi^{2} N(x, t)+\psi(x, t) \\
0 \leq \alpha \leq 1,0 \leq \beta \leq 1
\end{gathered}
$$

with initial conditions

$$
N(x, 0)=f(x), \quad \text { for } x \in R
$$

where $v$ is a diffusion coefficient, $\psi$ is constant which describes the nonlinearity in the system, and is a nonlinear function for reaction kinetics then there holds the following formula.

$$
\begin{aligned}
N(x, t)=\sum_{r=0}^{\infty} \frac{(-b)^{r}}{\sqrt{2 \pi}} \int_{-\infty}^{\infty} t^{\left(r+1-\alpha_{1}\right) \alpha+\alpha_{1}} f^{*}(k) \exp (-i k x) \times E_{\alpha-\beta, 1+\alpha r}^{r}\left(-a t^{\alpha-\beta}\right) d k \\
+\sum_{\mathrm{r}=0}^{\infty} \frac{(-b)^{r}}{\sqrt{2 \pi}} \int_{0}^{\mathrm{t}} \tau^{\alpha(r+1)-1} \int_{-\infty}^{\infty} \psi^{*}(k, t-\tau) \exp (-i k x) E_{\alpha-\beta, \alpha(r+1)}^{r+1} \\
\\
\left(-a \tau^{\alpha-\beta}\right) d k d \tau .
\end{aligned}
$$


Acknowledgments The first author is greatly acknowledges that this project is supported by Deanship of Scientific Research, College of Science Research Center, King Saud University.

\section{References}

1. Smoller, J.: Shock Waves and Reaction-Diffusion Equations, vol. 15. Springer, New York (1983)

2. Grindrod, P.: Patterns and Waves: The Theory and Applications of Reaction-Diffusion Equations. Clarendon Press, Oxford (1991)

3. Gilding, B.H., Kersner, R.: Traveling Waves in Nonlinear Diffusion-Convection Reaction. BirkhaeuserVerlag, Basel (2004)

4. Wilhelmsson, H., Lazzaro, E.: Reaction-Diffusion Problems in the Physics of Hot Plasmas. Institute of Physics Publishing, Bristol (2001)

5. Nicolis, G., Prigogine, I.: Self-Organization in Nonequilibrium Systems: From Dissipative Structures to Order Through Fluctuations. Wiley, New York (1977)

6. Henry, B.I., Wearne, S.L.: Fractional reaction-diffusion. Phys. A 276, 448-455 (2000)

7. Henry, B.I., Wearne, S.L.: Existence of Turing instabilities in a two-species fractional reaction-diffusion system. SIAM J. Appl. Math. 62, 870-887 (2002)

8. Jafari, H., Khalique, C.M., Nazari, M.: Application of the Laplace decomposition method for solving linear and nonlinear fractional diffusion-wave equations. Appl. Math. Lett. 24(11), 1799-1805 (2011)

9. Jafari, H., Momani, S.: Solving fractional diffusion and wave equations by modified homotopy perturbation method. Phys. Lett. A 370(5-6), 388-396 (2007)

10. Gupta, V.G., Sharma, B.: Application of Sumudu transform in reaction-diffusion systems and nonlinear waves. AMS 4(9), 435-446 (2010)

11. Manne, K.K., Hurd, A.J., Kenkre, V.M.: Nonlinear waves in reaction-diffusion systems: the effect of transport memory. Phys. Rev. E 61, 4177-4184 (2000)

12. Saxena, R.K., Mathai, A.M., Haubold, H.J.: Reaction diffusion systems and non linear waves. Astrophys. Space Sci. 305, 297-303 (2006)

13. Asiru, M.A.: Sumudu transform and the solution of integral equations of convolution type. Int. J. Math. Educ. Sci. Technol. 32(6), 906-910 (2001)

14. Belagecam, F.B.M., Karaballi, A.A.: Sumudu transform fundamental properties investigations and applications. Int. J. Appl. Math. Stoch. Anal. 2005, 1-23 (2005)

15. Belgacem, F.B.M.: Introducing and analysing deeper Sumudu properties. Nonlinear Stud. 13(1), 23-41 (2006)

16. Gupta, V.G., Shrama, B., Kiliçman, A.: A note on fractional Sumudu transform. J. Appl. Math. vol 2010, Article ID154189 (2010)

17. Eltayeb, H., Kilicman, A., Fisher, B.: A new integral transform and associated distributions. Integral Transforms Spec. Funct. 21(5), 367-379 (2010)

18. Kılıçman, A., Gadain, H.E.: On the applications of Laplace and Sumudu transforms. J. Frankl. Inst. 347(5), 848-862 (2010)

19. Mittag-Leffler, M.G.: Sur la nouvelle fonction E(x). Comptes Rendus Acad. Sci. Paris (Ser. II) 137, 554-558 (1903)

20. Wiman, A.: Ueber den Fundamentalsatz in der Theorie derFunctionen E(x). Acta Math. 29, 191-201 (1905)

21. Prabhakar, T.R.: A singular integral equation with generalized Mittag-Leffler function in the kernel. Yokohama Math. J. 19, 7-15 (1971)

22. Hilfer, R. (ed.): Applications of Fractional Calculus in Physics. World Scientific Publishing Company, Singapore (2000)

23. Hilfer, R., Luchko, Y., Tomovski, Ž.: Operational method for solution of the fractional differential equations with the generalized Riemann-Liouville fractional derivatives. Fract. Calc. Appl. Anal. 12, 299-318 (2009)

24. Liouville, J.: Mémoire sur quelques quéstions de géometrie et de mécanique, et sur un nouveau genre de calcul pour résoudre ces quéstions. J. École Polytech. 13(21), 1-69 (1832)

25. Caputo, M.: Elasticita e Dissipazione. Zanichelli, Bologna (1969)

26. Metzler, R., Klafter, J.: The random walks guide to anomalous diffusion: a fractional dynamics approach. Phys. Rep. 339, 1-77 (2000) 
27. Metzler, R., Klafter, J.: The restaurant at the end of the random walk: recent developments in the description of anomalous transport by fractional dynamics. J. Phys. A 37, R161-R208 (2004)

28. Goswami, P., Belgacem, F.B.M.: Fractional differential equation solutions through a Sumudu rational. Nonlinear Stud. 19(4), 591-598 (2012) 